\title{
Toxoplasmosis en Niños Tratados con Drogas de Efecto Inmunodepresor
}

\author{
Dres. Beatriz Gottlieb B. ${ }^{1}$; Hernán Reyes M. ${ }^{1}$; Peđro Herskovic L. ${ }^{2}$; \\ Erika Thiermann N. ${ }^{3}$; T. Méd. Myriam Lorca H. ${ }^{3}$; Berbeli Astorga L. 2 ; \\ Dres. Humberto del Pozo P.4; Victoria Beresi R.4; Juan Quintana B.4 Silvia Sanhueza M.4
}

\section{Toxoplasmosis in Children Treated witb Inmunodepressing Drugs}

\begin{abstract}
The frequency of infection with Toxoplasma gondii was studied in 129 children with various neoplastic diseases who were undergoing prolonged treatment with immunodepressant drugs. The indirect fluorescent antibody reaction was used to measure Toxoplasma antibodies, and titers equal to or greater than $1 / 16$ were considered diagnostic. Forty six patients $(35.7 \%)$ fell in this latter category; three of these $(2.3 \%)$ exhibited titers that suggested reactivation of the infection. The overall frcquency of Toxoplasma infection detected in our study goup is similar to that reported by other authors in normal Chilean children. Even though the risk of reactivation of the infection among our patients is low, its potentially severe, and even lethal, cansequences, make it recommendable to carry out serological surveys of these patients and to apply specific treatment when it is demonstrated.
\end{abstract}

La relación entre. inmunodepresión provocada por tratamiento prolongado por algunas drogas, (principalmente con las de acción antitumoral), y la reactivación o generalización de la Toxoplasmosis ha sido bien establecida por diversos autores $^{1-7}$. Sin embargo, la gran mayoría de estos estudios se refieren a pacientes adultos y aquellos que to hacen a nifios, corresponden sólo a relatos de casos aislados y no a estudios casuísticos prospectivos $^{8-9}$ Ahora bien, la magnitud de este riesgo en niños merece ser determinada, puesto que la infección por Toxoplasma gondii puede ser adquirida muy precozmente y alcanzar alta prevalencia ya en grupos de escasa edad. En nuestro pais la tasa de infección serológica en población normal adulta bordea el 50\%10-11, pero es importante destacar que estudios de seguimiento en nifos han permitido comprobar que ya entre los de más de 6-14 años de edad la frecuencia de infección es de $48 \% 12$.

En este trabajo se analizan los resultados obtenidos mediante estudio serológico para Toxoplasmosis en nifios sometidos a tratamiento prolongado con drogas inmunodepresoras, con el propósito de evaluar la frecuencia de dicha infección en este grupo de pacientes y la posible influencia del tratamiento inmunodepresor en cuanto al riesgo de adquirirla o reactivarla.

1 Unidad Parasitología, Depto. Salud Pública, Djv. Cs. Méd. Oriente, Universidad de Chile, Santiago.

2 Laboratorio de Referencia en Parasitología, Instituto de Salud Píblica de Chile, Santiago.

3 Unid. Parasitología. Depto. Prectínicos, Div. Cs. Méd. Occidente, Fac. Medicina, Universidad de Chile, Santiago.

4 Unid. Oncología, Serv. Pediatria, Hosp. J L. C. Macke nna., Santiago.

\section{PACIENTES Y METODOS}

De los pacientes que concurrieron al Policlínico de Oncologia del Hospital Luis Calvo Mackenna, Santiago, se estudiaron en forma sucesiva 129 niños (76 hombres y 53 mujeres) con edad comprendida entre 1 mes y 14 años: 12 eran menotes de 2 años, 53 entre 2 años 1 día y hasta 6 años y 64 eran mayores de 6 años y 1 día $\mathrm{y}$ hasta 14 años.

A cada uno de estos niffos se le hizo una ficha especial consignando la enfermedad de base, el tratamiento inmunodepresor que estaba recibiendo y los hallazgos del examen físico. Todos fueron sometidos a estudio serológico seriado mediante la reacción de-inmunofluorescencia indirecta (RIFI) para pesquisa de IgM antitoxoplas$\mathrm{ma}^{13-14}$. Las muestras de sangre respectivas fueron tomadas en el momento de ingreso a este estudio $y$, posteriormente, durante los controles clínicos a que debían acudir por su patología oncológica. En general, el segundo control serológico se realizó con un periodo mínimo de 15 dias, luego cada tres meses mientras duraba el tratamiento inmunodepresor $y$ cada 6 meses después.

Independientemente de este esquema general, se realizó control serológico para Toxoplasmosis en cada oportunidad en que algunos de estos pacientes presentaba un cuadro de características infecciosas que requirió hospitalización, en cualquiera de los controles clínicos a que estaba sometido.

Se consideró que RIFI era positiva para $T$. gondii cuando el título era igual o superior a $1 / 16^{11}$ y que existia un ascenso significativo de los títulos cuando aumentaban al cuádruple del valor basal. 
En algunos de estos pacientes fue necesario administrar tratamiento antitoxoplásmico. Para ello se empleó la asociación de Pirimetamina (1 $\mathrm{mg} / \mathrm{kg} / \mathrm{dia})$ y Sulfadiazina $(100 \mathrm{mg} / \mathrm{kg} / \mathrm{dia})$, por cuatro semanas ${ }^{10-11-15-17}$.

\section{RESULTADOS}

De 129 niños estudiados, 56 dieron resultados totalmente negativos con RIFI y 27 presentaron títulos de $1 / 4$, dando un total de $83(64,3 \%)$ interpretados como negativos, de acuerdo al cri. terio de lectura serológica indicado antes. Los 46 restantes $(35.7 \%)$ resultaron positivos: 33 $(25,6 \%)$ a título bajo, $9(7,0 \%)$ a título mediano y sólo $4(3,1 \%)$ a título alto (Tabla 1$)$.

Tabla 1

Reacción de Inmunofluorescencia Indirecta (título más alto) en 129 nir̄os inmmodeprimidos

\begin{tabular}{|c|c|c|c|c|}
\hline Título & No & n & TAL & $\begin{array}{l}\text { Interpretación } \\
\text { del Título }\end{array}$ \\
\hline $\begin{array}{l}\text { Negativo } \\
1 / 4\end{array}$ & $\begin{array}{l}56 \\
27\end{array}$ & 83 & 64.3 & Negativo \\
\hline $\begin{array}{l}1 / 16 \\
1 / 64\end{array}$ & $\begin{array}{l}21 \\
12\end{array}$ & 33 & 25.6 & Bajo \\
\hline $\begin{array}{l}1 / 256 \\
1 / 512 \\
1 / 1000\end{array}$ & $\begin{array}{l}2 \\
5 \\
2\end{array}$ & 9 & 7.0 & Mediano \\
\hline $\begin{array}{l}1 / 2000 \\
1 / 4000\end{array}$ & $\begin{array}{l}3 \\
1\end{array}$ & 4 & 3.1 & Alto \\
\hline
\end{tabular}

La comparación de estos resultados con los observados por Stagno y col. ${ }^{12}$ en niños aparentemente sanos, de edad comparable, no revela en general diferencias significativas (Tabla 2).

\section{Taba 2.}

Comparación de porcentajes de seropositivos por edad entre niños inmunodeprimidos y niños normales

\begin{tabular}{lcccccc} 
& \multicolumn{3}{c}{ INMUNODEPRIMIDOS } & \multicolumn{3}{c}{ NORMALES (*) } \\
$\begin{array}{l}\text { Edad } \\
\text { (años) }\end{array}$ & Total & $n$ & $\%$ & Total & $n$ & $\%$ \\
& & & & & & \\
1 & 3 & 0 & 0.0 & 50 & 1 & 2.0 \\
$1-2$ & 7 & 3 & $(0)$ & 50 & 1 & 10.0 \\
$2-4$ & 36 & 9 & 25.0 & 50 & 11 & 22.0 \\
$4,1-6$ & 21 & 7 & 33.3 & 50 & 16 & 32.0 \\
$6,1-14$ & 62 & 27 & 43.5 & 50 & 24 & 48.0
\end{tabular}

(*) Según datos de Stagno y col. 1973.

(*) No calculado por escaso número de casos estudia. dos.
La Tabla 3 muestra la enfermedad de base que padecían estos niños y que motivó el tratamiento inmunodepresor. Como se observa, 79 casos $(61,2 \%)$ fueron debidas a Leucemias y Linfomas.

Tabla 3.

Toxoplasmosis en niños con tratamiento inmunodepresor serología positiva $y$ enfermedad de base

Enfermedad de base

No casos

Positivos

Leucemia Linfoblástica

aguda

$30 \quad 10$

Leucemia Mieloblástica

aguda

Linfoma No Hodgkin

Linfoma Hodgkin

Sarcoma

Tumor de Wilns

Histiocistosis

Carcinoma

Neuroblastoma

Retinobjastoma

Varios

$\begin{array}{rr}9 & \\ 27 & 5 \\ 13 & 10 \\ 14 & 5 \\ 10 & 4 \\ 7 & 3 \\ 5 & 2 \\ 6 & 1 \\ 2 & 1 \\ 6 & 2\end{array}$

En la Tabla 4 se comparan los títulos para Toxoplasmosis entre los niños con Leucemia o Linfomas y la población normal. Tampoco se observan diferencias significativas. De los 12 casos con títulos igual o superior a1/512, 6 correspondieron a pacientes con Linforna, 3 a Leucemias, 2 a Sarcoma y 1 a Turmor de Wilms.

\section{Tabla 4.}

Toxoplasmosis en niños con tratamiento inmunodepresor.

Comparación de títulos serológicos en pacientes con Leucemia, Linfoma y Población Normal.

\begin{tabular}{|c|c|c|c|c|c|}
\hline \multirow[t]{2}{*}{ Título } & \multicolumn{2}{|c|}{ Letsemias (n 39) } & \multicolumn{2}{|c|}{ Linforna (n 40) } & $\begin{array}{l}\text { Pobl. } \\
\text { Nomal (*) }\end{array}$ \\
\hline & n & 86 & $\mathrm{n}$ & $\%$ & $\%$ \\
\hline Negativo & 24 & 61.5 & 25 & 62.5 & 64.3 \\
\hline Bajo Mediano & 13 & 33.3 & 12 & 30.0 & 26.3 \\
\hline Alto & 2 & 5.1 & 3 & 7.5 & 9.3 \\
\hline
\end{tabular}

(*) Según datos de Stagna y col. (1973).

Aunque se estudió la evolución de los títulos serológicos en todos los niños, la Tabla 5 muestra solamente los resultados de los casos en que, como mínimo, pudo realizarse 4 controles sero. lógicos sucesivos. Puede observarse que en 40 $(85,1 \%)$ no tubo variación en los títulos y de los $7(17,5 \%)$ en que esta ocurrió, sólo $3(7,5 \%)$ tuvieron un ascenso significativo. Estos 3 últimos pacientes (Tumor de Wilms grado IV con metás- 
tasis pulmonar, Linfoma No Hodgkin y Linfoma Hodgkin, respectivamente) fueron sometidos a tratamiento contra la toxoplasmosis. En sólo uno de estos casos se observó descenso significativo de los títulos luego del tratamiento, en otro no hubo variación y en el tercero los títulos siguieron ascendiendo, hasta el fallecimiento del paciente.

Tabla 5 .

Toxoplasmosis en niños con tratamiento inmunodepresor.

Evolución de títulos serológicos en

47 niños con al menos 4 determinaciones

\section{Evolución Serológica}

Persiste negativo

Persiste pasitivo

Ascenso

Descenso

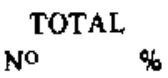

$40 \quad 85.1$
13

\section{DISCUSION}

El primer propósito de este estudio fue evaluar la frecuencia de la Toxoplasmosis en un grupo representativo de pacientes infantiles que estaban siendo sometidos a tratomiento prolongado con drogas de efecto inmunodepresor, por una enfermedad oncológica. Se ha postulado que en pacientes inmunodeprimidos existe mayor probabilidad para adquirir la Toxoplasmosis. Sin embargo, los resultados obtenidos no confirman lo anterior, pues los niños estudiados presentan porcentajes de infección, por grupos de edad, del todo comparables con los encontrados en población infantil normal de nuestro medio ${ }^{12}$.

Tal vez ello se deba a que, en realidad, ambos grupos ti:nen iguales posibilidades de infectarse, por compartir un ambiente similar. Recordemos que el parásito es infectante en cualquiera de las formas evolutivas y los niños pueden adquirirlo precozmente al ingerir agua, frutas y verduras contaminadas con heces de gato, al jugar con tierra o arena igualmente contaminada, por sus contactos directos con estos animales, al ingeric carne cruda o con cocción insuficiente, por transfusiones sanguíneas, o por infección congénita.

Al respecto, nuestra serie no incluye casos de infecciones congénitas pues sólo 3 niños tenian menos de 1 año y ninguno serología positiva.

Otra explicación posible es que los estudios de quienẹs afirman que hay mayor riesgo de infec- ción en pacientes inmunodeprimidos han sido realizados en centros donde se usan recursos terapéuticos poco empleados en Chile, como transfusiones leucocitarias, cuyo papel en la transmisión iatrogénica de la Toxoplasmosis ha sido demostrado 18 -19.

El segundo propósito de este trabajo fue evaluar la posible influencia del tratamiento inmunodepresor en cuanto al peligro de reactivar la Toxoplasmosis. Recordemos que la infección es habitualmente asintomática 10-11-15 y sólo en un porcentaje pequeño puede provocar patología importante. Se acepta que esto se debe al desartollo de inmunidad protectora humoral y celular, a pesar de que esta defensa es insuficiente para eliminar totalmente la infección del huésped, pues el parásito puede eludir su efecto introduciéndose en células ${ }^{20-26}$, y permanecer alli en forma de quistes de reproducción lenta. Persiste asi, como infección latente, mientras la defensa se mantenga, lo que depende -en parte- de un adecuado nivel de anticuerpos, medidos a través de reacciones serológicas. Si la defensa se altera por inmunodepresión (iatrogénica, entre otros factores) puede ocurrir la reactivación y la diseminación de la infección, con serio peligro para la vida del paciente, pues, en tales casos, el parásito parece tener especial predilección por el sistema nervjoso central. Esto podría deberse a que los anticuerpos no logran llegar allí, en cantidad adecuada, por la barrera hematoencefálica, lo cual, asociado al efecto inmunodepresor, que también interfiere la inmunidad celular, facilitaría dicha localización ${ }^{27-33}$.

Del total de pacientes estudiados, sólo 3 (2.3\%) presentaron ascenso significativo de sus títulos luego de iniciar el tratamiento de la enfermedad de base (Tabla 5), 10 que puede serinterpretado como un indice de reaetivación: Estos casos fueron tratados 'con la asociación Pirimetamina-Sulfadiazina, como se 'indicó antes, para evitar una diseminación masiva, pero apenas en uno de ellos hubo descenso significativo de la curva serológica, en otro no se modificó y en el último siguió ascendiendo hasta la muerte del paciente. Es decir, según los resultados de este estudio, el riesgo de reactivación por inmunodepresión apareció en baja proporción, pero no debemos olvidar que puede originar problemas severos en los pacientes afectados, o, al menos, agravando su condición.

Es importante estar alertas frente a la posible aparición de complicaciones infecciosas en pacientes que deben ser sometidos a tratamiento prolongado con drogas de efecto inmunodepresor. Hay que tener presente que el cuadro clínico respectivo se agrega al de la enfermedad de base 
y, por otra parte, puede estar alterado por el mismo efecto inmunodepresor. Sólo un alto indice de sospecha permitirá, entonces, establecer oportunamente el diagnóstico y tratamiento respectivo.

En la Toxoplasmosis estas consideraciones tienen gran valor, no solamente por la frecuencia con que esta infección puede ser encontrada en tales pacientes - prácticamente en la misma proporción que en la población infantil normal, como to hemos constatado en este estudio-, sino también porque su cuadro clínico no es patognomónico y puede adquirir caracteristicas polimorfas al asociarse al de la enfermedad de base. Existe el riesgo de compromiso cerebral y extracerebral y dar, según su ubicación, síntomas y signos de meningoencefalitis, encefalopatía difusa, tumor cerebral, miocarditis, hepatitis por T. gondii y otros $6-31-34-36$.

Seria aconsejable hacer una determinación serológica para Toxoplasmosis antes de iniciar tratamiento con inmunodepresores, para tener un nivel de referencia previo, y luego, controles seriados, porque es importante observar la evolución de los títulos de anticuerpos, si permanecen estables o se modifican. Al respecto, conviene tener en cuenta que en pacientes inmunode. primidos, tal vez sea necesario no orientarse por los criterios clásicos de la variación del título (significativo si se eleva al cuádruple) sino, incluso, con ascensos menores. En estos últimos casos, creemos aconsejable repetir la reacción serológica en un plazo menor al habitual (15 días), para apresurar la confirmación del diagnóstico. Por supuesto, que este control serológico hay que efectuarlo cada vez que presente un cuadro infeccioso importante de etiología no precisada principalmente si la ubicación es del sistema nervioso central.

Los métodos serológicos son actualmente los más empleados para el diagnóstico de la Toxoplasmosis ${ }^{10-11-15}$. Clínicamente se ha aceptado que la reacción patrón es la de Sabin y Fledman, pero por compleja y costosa ha sido reemplazada por 1a reacción de Inmunofluorescencia Indirecta (RIFI), que presenta resultados equivalentes ${ }^{3 a-42}$. La determinación de la IgM especifica mediante RIFI puede ayudar también en el djagnóstico de la infección ${ }^{\mathbf{1 4}-43}$, pero solamente cuando es positiva, puesto que si es negativa no permite descartarla. En los pacientes de esta serie la IgM específica resultó positiva en los primeros dos casos tratados, en el segundo se hizo negativa, lo que podría atribuirse al efecto del tratamiento antitoxoplasmósico como también a la extinción natural de la $\mathrm{IgM}$.

El tratamiento oportuno es salvador. Así en una serie de los casos de Toxoplasmosis en inmunodeprimidos, 26 fueron tratados $y_{1}$ de ellos, 16 mejoraron; el resto no.recibió tratamiento porque no se hizo el diagnóstico y, de ellos, sólo 2 sobrevivieron. ${ }^{20}$

En surna, este trabajo demuestra que la frecuencia de infección por Toxoplasmosis en inmunodeprimidos es similar a la población infantil normal y que existe riesgo de reactivación con el tratamiento inmunodepresor. Se aconseja control serológico seriado de estos niños y eventual tratamiento específico, si se demuestra infección aguda.

\section{RESUMEN}

La Toxoplasmosis puede constituir una infección grave $e$ incluso mortal en inmunodeprimidos.

Este trabajo analiza la frecuencia de esta infección, medjante estudio serológico, ел 129 niños sometidos a tratamiento prolongado con drogas inmunodepresoras, demostrando que es similar a la población infantil normal.

Se demuestra que existe un riesgo bajo $(2,3 \%$ de reactivación de la infección por $T$. gondii con el tratamiento inmunodepresor aunque no despreciable dado las consecuencias de esta infección.

Se aconseja control serológico seriado de estos nifios y eventual tratamiento especifico, si se demuestra infección aguda.

\section{REFERENCIAS}

1 Cohen S.N.i Toxoplasmosis in patients receiving immunosuppressive therapy. JAMA, 211: 657, 1970.

2 Vietzke W.N. et al.: Toxoplasmosis complicating maingnancy. Experience at the National Cancer Institute. Cances, 5: 816, 1968.

3 Vietzke W.M. et al.: Toxoplasmosis complicating malignancy. Cancer, 21 : 816, 1968.

4 Glesson T.M., Hamlin W.B.: Disseminated Toxoplasmosis in the compromised host. Arch. Interm. Med. 134: 1059, 1974.

5 Frenkel J.K. et al.: Immunosuppression and Toxoplasmic encephalitis. Human Pathology, 6: 97, 1975.

6 Ruskin I., Remington J.S.: Toxoplasmosis in the compromised host. Ann. Intern. Med., 84: 193, 1976.

7 Hafizi A., Modabber F.Z: Effect of cyclophosphamide en Toxoplasma gondif infection: reversal of the effect by passive immunization. Clin. Exp. Immunol., 33: 389, 1978.

${ }^{8}$ Hoof C. et al: Leucemie et Toxoplasmose. Arch. Franc. Ped. 19: 1201, 1962.

9orella R., Webster R.G.: The immunosssupressive cffects of longterm combination chemotherapy in children with acute Leukemia in remission. Cancer Research, 31: 420, 1971.

10 At ́as A., Thiermann E.: Toxoplamosís (En "Parasitología Clínica" Atías y Neghme Ed., Capítulo 28)., 
Ed. Intermédica, Buenos Aires, Argentina: 231, 1979.

11 Apr W., et al.: Toxoplasmosis. Monografía Científica 23. Universidad de Chile, 1973.

12 Stegno S., Thiermann E.: Acquisition of Toxoplasma infection by children in a developing country. Bull. Wid. Hlth. Org $49: 627,1973$.

13 Conrarh $T$.: Handbook of microtiter procedures. Cambridge Mass. Dynateech Corporation: 321, 1972.

14 Remington J.S. et al.: IgM antibodies in acute Toxoplasmosis. J. Lab. Clin. Med., 71: 855, 1968.

15 Schmidt G., Roberts L.S.: Toxoplasma (En "Foundations of Parasitology". Capítulo 7), Ed. Mosby $12 \mathrm{~B}, 1981$

16 Frenkel J.K. et al.: Acute Toxoplasmosis effective treatment with 'Pyrimethamine, Sulfadiazine e Leucovorin calcium and yeast. JAMA, 173: 1471, 1960.

17 Helmer R.E.: Hazard of folinic acid with pyrimethamine and sulfadiazine. Ann. Inter. Med., 82: 1975.

18 Roth J.A. et al.: Fatal recurrent Toxoplasmosis in a patient initially infected in a leukocyte transfusion. Am. J. Clin. Path. 56: 601, 1971.

19 Siegel S.E. et al.: Transmission of Toxoplasmosis by leukocyte transfusion. Blood, 37: 388, 1971.

20 Frenkel J.K.: Adoptive immunity to intracellular infection. J. Immunol. 98: 1309, 1967.

21 Frenkel J.X., Wilson H.R.: Effects of radiation on specific cellular immunities: Besnoitiosis and herpes virus infection of hamsters. $J$. Infect. Dis. 125:216, 1972.

22 Hoff R., Frenkel J.K.: Cell-mediated immunity against Besnoitia and Toxoplasma in specifically and cross - immunizated hamsters and in culture. $J$. Exp. Med., 139: 560, 1974.

${ }^{23}$ Hoff $H$. et ala Infection of congenitally athymic (nude) mice with Toxoplasma gondii. Ann. Microbiolog. B., 127: 503, 1976.

24 Lindberg R.E., Frenkel $J . K .:$ Toxoplasmosis in nude mice. J. Parasitolog. 63: 219, 1977.

25 Lindberg R.E., Frenkel J.K.: Cellulas immunity to Toxoplasma and Besnoitia in hamsters: specificity and the effects of cortisol. Infect. Immun. 15: 855 , 1977.

26 Frenkel J.K. et al.: Immune competence in a patient with Hodgkin's disease and relapsing Toxoplasmosis. Infection 6: 84, 1979.

27 Cheever A.W. et at. Necrotizing toxoplasmic encephalitis and herpetic pneumonia complicating treated Hodgkin's disease. New Engl. J. Med. 272: 26,1965 .
28 Theologides A. et al.: Cerebral Toxoplasmosis in multiple myeloma. Ann. Intern. Med., 64: 1071, 1966.

29 Chalok NR.: Toxoplasmosis of the central nervous system in the adult Arch. Pathol, 89: 337, 1970.

30 Bamford C.R.- Toxoplasmosis mimicking a brain abscess in an adult with treated scleroderma. Neurology, 25: 343, 1975.

31 Nicholson D.H., Wolclok E.: Ocular Toxoplasmosis in an adult receiving long-term corticosteroid therapy. Arch. Ophthalmol., 94: 248, 1976.

32 Mc. Leod. T. et al.: Toxoplasmosis presenting as brain abcesses. Diagnosis by computarized tomography and cytology of aspirated purulent material. Am. J. Med., 67: 711, 1979.

33 Conley et al.: Toxoplasma gondit infection of the nervous system. Human Pathology, 12 (B): 690, 1981.

34 Wertlake P.T. Winter T.S.: Fatal Taxoplasma myocarditis in an adult patient with acute lymphocy tic leukemia. N. Engl. J. Med., 237: 438, 1965.

35 Reynolds ES: et al.: Generalized Toxoplasmosis following renal transplantation. Arch. Inter., Med., 118: 401, 1966.

36 Anthony C.W.: Disseminated Toxoplasmosis in a liver transplant patient. J. Am. Med. Wom. Assoc., 27: 601, 1972.

37 Camargo M.E.: Improved technique of immunofluorescense for serological diagnosis of Toxoplasmosis Rev. Int. Med. Trop. S. Paulo, 6: 117, 1964

38 Stogno S., Thiemmenn E.: La Técnica de la insunofluorescencia indirecta en el diagnóstico, serológico de la Toxoplasmosis. Pediatría, 12: 124, 1969.

39 Stagno S., Thiermann E.: Valor de la inmunofluorescencia indirecta en el diagnóstico serológico de la Toxoplasmosis aguda. Bol. Chil. Parasit., 25: 9, 1970.

40 Stagno S. ef al.: Comparación de las reacciones de Sabin y Feldman e inmunofluorescencia indirecta para Toxoplasmosis en 1263 sueros humanos. Bol. Chile. Parasit., 25: 102, 1970.

41 Camargo M.E. et al:: Diagnosis information from serological test in Human Toxoplasmosis: A com parative study of hemaglutination, complement fixation, IgG immunofluorescente test in 3752 serum samples. Rev. Inst., Med. Trop. S. Paulo, 18 : 215,1976 .

42 Tello $P$., Sonhueza J.t Evaluación de un test de inmunofluorescencia indirecta en el diagnóstico serológico de la Toxoplasmosis. Bol. Chile. Parasit. $33: 12,1978$.

43 Frenkel J.K. et al.: Immune competence in a patient with Hodgkin's disease and relapsing Toxoplasmosis. Infection, 6: 84, 197. 